\title{
Hematoma iatrogênico simulando lesão tumoral intradural lombar
}

\author{
José Luís Monteiro Alves ${ }^{1}$, José Nubélio Cristovão Duarte', \\ José Gustavo Bento Soares², Armando Manuel Tavares Rocha² \\ Serviço de Neurocirurgia de Coimbra, Centro Hospitalar e Universitário de Coimbra, Coimbra, Portugal.
}

\section{RESUMO}

Em um quadro clínico de lombociatalgia, o diagnóstico diferencial engloba uma variedade de possíveis etiologias. Descreve-se um caso de um doente do sexo feminino, com história de lombociatalgia incapacitante relacionada com hematoma intradural, consequência de punção lombar e drenado após laminectomia lombar. São revistas as várias séries descritas na literatura, assim como são discutidos os diferentes fatores de risco, quadros clínicos e estratégias de prevenção e tratamento.

\section{PALAVRAS-CHAVE}

Coluna vertebral, doença iatrogênica, punção espinal, dor lombar.

\section{ABSTRACT}

latrogenic hematoma mimicking lumbar intradural tumoral lesion

In the presence of lumbar radicular pain, the differential diagnosis includes several possible etiologies. Here is described a case of a female patient with incapacitating low back pain and lumbar radicular pain caused by an intradural hematoma, consequence of lumbar puncture, surgically drained after laminectomy. The several series in the literature describing these cases are revised, and the different risk factors, clinical pictures, prevention and treatment strategies are discussed.

\section{KEYWORDS}

Spine, iatrogenic disease, spinal puncture, low back pain.

1 Interno do Internato Complementar de Neurocirurgia, Serviço de Neurocirurgia, Centro Hospitalar e Universitário de Coimbra, Coimbra, Portugal.

2 Neurocirurgião, Serviço de Neurocirurgia, Centro Hospitalar e Universitário de Coimbra, Coimbra, Portugal. 


\section{Introdução}

Hematomas intradurais lombares são uma ocorrência rara, usualmente associados a traumatismos ou discrasias sanguíneas, podendo ter causa iatrogênica ou eventualmente ser de natureza espontânea. Os autores reportam um raro caso clínico de lombociatalgia bilateral no seguimento de um hematoma intradural lombar iatrogênico, causado por punção lombar diagnóstica quando do estudo de patologia craniana não relacionada. A literatura relevante é revista e são discutidos os diferentes achados clínicos, etiologia e patogênese.

\section{Caso clínico}

Doente do sexo feminino, 46 anos de idade, sem antecedentes pessoais significativos, seguida em consulta de Neurologia no contexto de vertigens posicionais e perturbações do equilíbrio, efetuou ressonância magnética (RM) cranioencefálica, com achados descritos como “(...) possíveis lesões desmielinizantes/vasculares num quadro de vasculite". Os restantes exames complementares, incluindo punção lombar, foram inconclusivos. Ainda em internamento, após instalação de um quadro de lombociatalgia bilateral de progressiva intensidade, estudo posterior por RM da coluna vertebral demonstrou uma "formação de provável natureza expansiva intradural que se prolonga desde o bordo inferior de L4 até ao bordo superior de S1 (Figura 1) - provável lesão

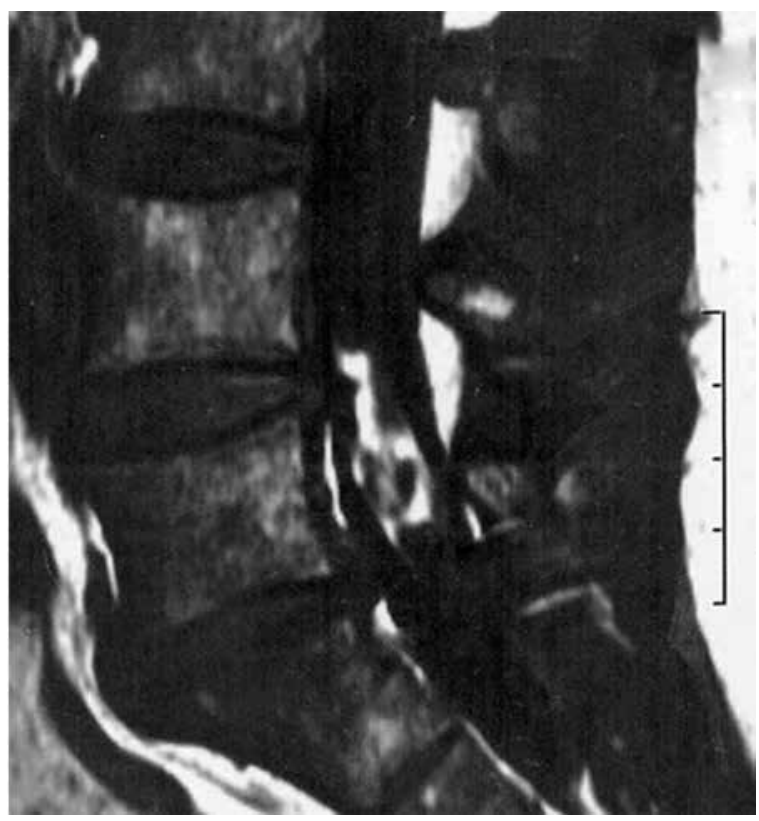

Figura 1 - RM de coluna lombar, ponderação T1, imagem em corte axial de lesão intradural, com realce ao contraste. com componente lipomatoso associado" (Figura 2). Foi submetida à intervenção cirúrgica eletiva 13 dias após a punção lombar, tendo-se procedido à laminectomia de L5, à durotomia e à drenagem da lesão, que se revelou um hematoma intradural lombar, organizado e com efeito de massa significativo. A evolução pós-operatória foi satisfatória, com ausência de sintomas ou sequelas no seguimento em longo prazo.

\section{Discussão}

Na presença de um quadro de lombalgia e/ou ciatalgia, a lista de possíveis diagnósticos diferenciais é vasta: hérnia/protusão discal; processos degenerativos de causas congênitas; estenose canalar ou listesis lombar; processos infecciosos (espondilodiscite, herpes-zóster); tumores ósseos da coluna lombar; metastização; processos neoplásicos dos tecidos moles no trajeto do nervo (intra-abdominais, coxa); processos inflamatórios (bursite, miosite); síndrome do piriforme, neuropatias periféricas e outros. Como causa específica de lombalgia/ciatalgia, apesar de raros, são ainda de considerar processos hemorrágicos intracanalares espontâneos ou de natureza iatrogênica. Primeiro descritos por Jackson em $1869,{ }^{1}$ os hematomas do ráquis constituem uma entidade patológica multifatorial rara, mas merecedora

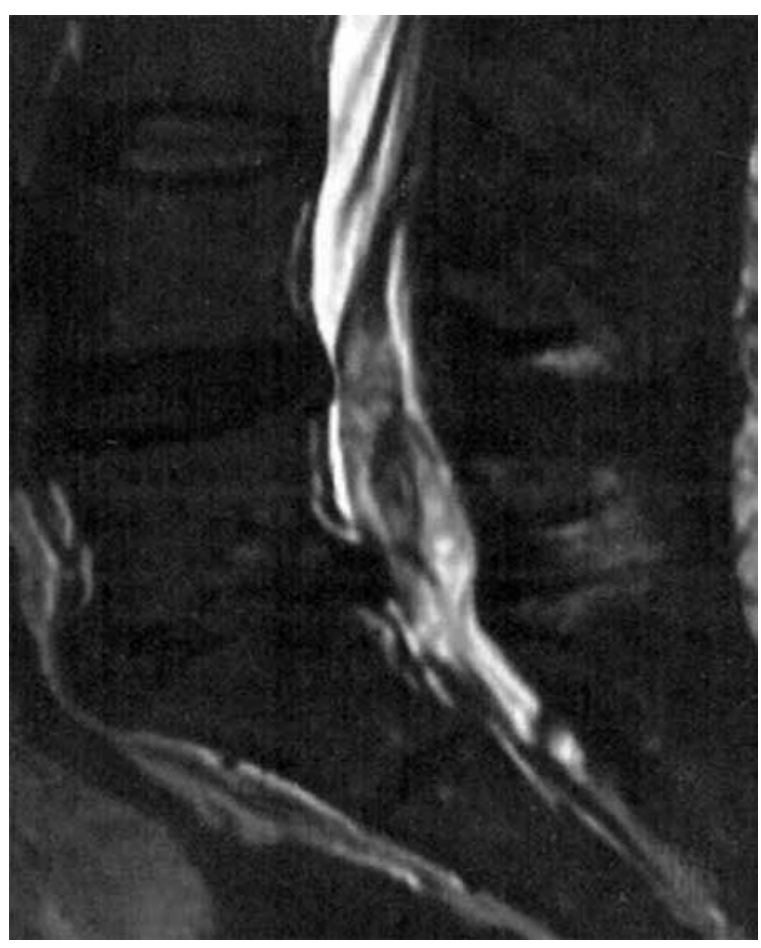

Figura 2 - RM de coluna lombar, ponderação T2, imagem em corte axial de lesão intradural. 
de atenção em quadros clínicos sugestivos. Estudos de imagem [tomografia computadorizada (TC) e ressonância magnética $(\mathrm{RM})$ ] pós-operatória precoce em doentes submetidos a cirurgia de hérnia discal e/ou procedimento descompressivo demonstraram hematomas epidurais assintomáticos em todos os doentes submetidos à microdiscectomia, ${ }^{2,3}$ nomeadamente nos procedimentos multiníveis, com $43 \%$ registrando volume significativo e estendendo-se até ao saco dural. No caso das punções lombares ou anestesia epidural, as múltiplas tentativas de punção constituem por si só, por causa do traumatismo repetido, um fator de agravamento do risco. Alguns autores ${ }^{4-6}$ referem pequenas hemorragias do ligamentum flavum como fatores causais. Lawton et al. ${ }^{7}$ referem uma taxa de incidência de $0,1 \%$ de hematomas epidurais sintomáticos após punção lombar.

No caso dos hematomas ditos intradurais, a sua incidência é ainda menor - Kasliwal et al. ${ }^{8}$ referem 72 casos descritos até 2010, a maioria dos quais associados a anomalias da coagulação ou terapêutica anticoagulante. ${ }^{9}$ A revisão por Singh et al. ${ }^{10}$ faz referência a $19 \%$ dos casos associados à discrasia sanguínea (leucemia, hemofilia, trombocitopenia ou outros) e $35 \%$ dos casos em relação com terapêutica anticoagulante. Por outro lado, em $47 \%$ dos casos, a iatrogenia por punção lombar era o fator causal major (em 33\% dos casos estava associada a algum grau de alteração da coagulação). A sua frequente relação com terapêuticas trombolíticas ou anticoagulantes, nomeadamente heparinas de baixo peso molecular em altas doses, confirma a importância da suspensão, se possível, desse gênero de intervenção farmacológica, na previsão de um dos procedimentos anteriormente referidos. ${ }^{11}$ Contudo, a terapêutica anticoagulante pode por si só induzir hematomas espontâneos. Esses podem igualmente estar associados a lesões hipervascularizadas/malformações vasculares (nomeadamente hemangiomas), lesões neoplásicas, discrasias sanguíneas, doenças hepáticas ou autoimunes, processos infecciosos, traumatismos prévios ou até mesmo gravidez. ${ }^{8,12,13}$ Outros possíveis fatores de risco são: terapêutica pré-operatória com anti-inflamatórios não esteroides, idade avançada, grupo $\mathrm{Rh}+$, valores de hemoglobina $<10 \mathrm{~g} / \mathrm{dl}$, valores alterados de INR (international normalized ratio). ${ }^{14-16}$ Neste caso específico, não se verificava nenhum desses fatores de risco. A inserção da agulha foi efetuada na linha média, técnica considerada como tendo maior taxa de sucesso (menos tentativas, menor risco) em relação à técnica paramediana.

A sua patogênese é ainda controversa: a ausência de veias em ponte no espaço subdural pode explicar, segundo Bortolotti et al. ${ }^{13}$ a raridade desse fenômeno. Porém, Durupt et al. ${ }^{17}$ sublinham ainda a possibilidade de punção da artéria radiculomedular ou até mesmo da artéria de Adamkiewicz como elemento causal. Outras teorias relevam o papel dos vasos do espaço subaracnói- deo, passíveis de ruptura mediante variações bruscas de pressão torácica/abdominal, com posterior drenagem para o espaço subdural e formação de hematoma. ${ }^{18,19}$

A maioria dos hematomas subdurais, nomeadamente os iatrogênicos, é assintomática. Porém, nos raros casos com repercussão clínica, a sintomatologia é variada, desde lombalgias moderadas a um quadro ciatálgico de intensidade crescente, podendo progredir para plegia flácida, perturbações sensitivas e/ou alterações esfincterianas. Na presença de paraparésia/paraplegia de instalação rápida e progressiva $(\leq 0,1 \%$ dos casos $),{ }^{10} \mathrm{a}$ intervenção cirúrgica de emergência é mandatória, sob o risco de sequelas neurológicas severas. A intervenção precoce nas primeiras $8 \mathrm{~h}$, segundo Vandermeulen et al. ${ }^{20} \mathrm{e}$ Segabinazzi et al. ${ }^{21}$ ou nas primeiras $12 \mathrm{~h}$, segundo Sinclair et al., ${ }^{22}$ aumenta as hipóteses de recuperação neurológica do défice provocado pela compressão radicular por um hematoma, ${ }^{23}$ possivelmente devido à reversão de fenômenos isquêmicos locorregionais. Börm et al. ${ }^{24}$ referem ainda uma forte relação entre o status neurológico inicial e o outcome final. Como fator de mau prognóstico, é também apontada a existência de um componente subaracnóideo. ${ }^{18} \mathrm{Na}$ literatura, há referência a um único caso de recuperação significativa após cirurgia realizada mais de $24 \mathrm{~h}$ depois da instalação do défice neurológico. ${ }^{25}$

O diagnóstico diferencial ganha importância no enquadramento dos achados imagiológicos com a história pregressa do doente. O estudo por RM, nomeadamente na distinção com um hematoma epidural, ${ }^{6,26,27}$ e provas de coagulação são imprescindíveis. Porém, a ausência de alterações de coagulação não exclui esse diagnóstico, nomeadamente nos casos de hematoma espontâneo. ${ }^{22,28,29}$

$\mathrm{O}$ achado peroperatório descrito - hematoma intradural lombar iatrogênico, mimetizando lesão tumoral - é uma das possíveis complicações da punção lombar e deverá ser tido em conta, mesmo em doentes sem fatores de risco, como no caso clínico relatado. A sua localização pode resultar numa morbilidade significativa, implicando possíveis sequelas evitáveis com a detecção precoce do problema.

\section{Conflito de interesses}

Os autores declaram não haver conflito de interesses na realização deste trabalho.

\section{Referências}

1. Jackson R. Case of spinal apoplexy. Lancet. 1869;2:5-9. 
2. Kotilainen E, Alanen A, Erkintalo M, Valtonen S, Kormano M. Magnetic resonance image changes and clinical outcome after microdiscectomy or nucleotomy for ruptured disc. Surg Neurol. 1994;41(6):432-40.

3. Sokolowski MJ, Garvey TA, Perl J $2^{\text {nd }}$, Sokolowski MS, Cho W, Mehbod AA, et al. Prospective study of postoperative lumbar epidural hematoma: incidence and risk factors. Spine (Phila Pa 1976). 2008;33(1):108-13.

4. Lunardi P, Mastronardi L, Lo Bianco F, Schettini G, Puzzilli F. Chronic spontaneous spinal epidural hematoma simulating a lumbar stenosis. Eur Spine J. 1995;4(1):64-6.

5. Minamide A, Yoshida M, Tamaki T, Natsumi K. Ligamentum flavum hematoma in the lumbar spine. J Orthop Sci. 1999;4(5):376-9.

6. Albanese A, Braconi A, Anile C, Mannino S, Sabatino G, Mangiola A. Spontaneous haematoma of ligamentum flavum. Case report and literature review. J Neurosurg Sci. 2006;50(2):59-61.

7. Lawton MT, Porter RW, Heiserman JE, Jacobowitz R, Sonntag VK, Dickman CA. Surgical management of spinal epidural hematoma: relationship between surgical timing and neurological outcome. J Neurosurg. 1995;83(1):1-7.

8. Kasliwal MK, Agrawal D, Sharma BS. Spinal subdural haematoma following traumatic lumbar puncture in a patient with normal coagulation profile. Pan Arab J Neurosurg. 2010;2:96-98.

9. Bills $D C$, Blumbergs $P$, North JB. latrogenic spinal subdural haematoma. Aust N Z J Surg. 1991;61(9):703-6.

10. Singh DK, Chauhan M, Gupta V, Chopra S, Bagaria HR. Spinal subdural hematoma: a rare complication of spinal anesthesia: a case report. Turk Neurosurg. 2008;18(3):324-6.

11. Gurkanlar D, Acikbas C, Cengiz GK, Tuncer R. Lumbar epidural hematoma following lumbar puncture: the role of high dose LMWH and late surgery. A case report. Neurocirugia (Astur). 2007;18(1):52-5.

12. Gutterman P. Acute spinal subdural hematoma following lumbar puncture. Surg Neurol. 1977;7(6):355-6.

13. Bortolotti C, Wang H, Fraser K, Lanzino G. Subacute spinal subdural hematoma after spontaneous resolution of cranial subdural hematoma: causal relationship or coincidence? Case report. J Neurosurg. 2004;100(Suppl 4):372-4.

14. Kou J, Fischgrund J, Biddinger A, Herkowitz H. Risk factors for spinal epidural hematoma after spinal surgery. Spine (Phila Pa 1976). 2002;27(15):1670-3.

15. Awad JN, Kebaish KM, Donigan J, Cohen DB, Kostuik JP. Analysis of the risk factors for the development of postoperative spinal epidural haematoma. J Bone Joint Surg Br. 2005;87(9):1248-52.

16. Aono H, Ohwada T, Hosono N, Tobimatsu H, Ariga K, Fuji $\mathrm{T}$, et al. Incidence of postoperative symptomatic epidural hematoma in spinal decompression surgery. J Neurosurg Spine. 2011;15(2):202-5.
17. Durupt S, Durieu I, Nove-Josserand R, Raynal C, Levrat $\mathrm{R}$, Vital Durand D. A rare complication of lumbar puncture: the spinal subdural hematoma. Rev Med Interne. 2000;21(2):199-200.

18. Domenicucci M, Ramieri A, Ciappetta P, Delfini R. Nontraumatic acute spinal subdural hematoma: report of five cases and review of the literature. J Neurosurg. 1999;91(Suppl 1):65-73.

19. Miller DR, Ray A, Hourihan MD. Spinal subdural haematoma: how relevant is the INR? Spinal Cord. 2004;42(8):477-80.

20. Vandermeulen EP, Van Aken H, Vermylen J. Anticoagulants and spinal-epidural anesthesia. Anesth Analg. 1994;79(6):1165-77.

21. Segabinazzi D, Brescianini BC, Schneider FG, Mendes FF. Conservative treatment of hematoma after spinal anesthesia: case report and literaturereview. Rev Bras Anestesiol. 2007;57(2):188-94.

22. Sinclair AJ, Carroll C, Davies B. Cauda equina syndrome following a lumbar puncture. J Clin Neurosci. 2009;16(5):714-6.

23. Kebaish KM, Awad JN. Spinal epidural hematoma causing acute cauda equine syndrome. Neurosurg Focus. 2004;16(6):e1.

24. Börm W, Mohr K, Hassepass U, Richter HP, Kast E. Spinal hematoma unrelated to previous surgery: analysis of 15 consecutive cases treated in a single institution within a 10-year period. Spine (Phila Pa 1976). 2004;29(24):E555-61.

25. Russell NA, Mangan MA. Acute spinal cord compression by subarachnoid and subdural hematoma occurring in association with brachial plexus avulsion. Case report. J Neurosurg. 1980;52(3):410-3.

26. Graftieaux JP, Scavarda D, Gomis P, Léon A, Rousseaux R. Bilateral sciatica: a predictive sign of extradural hematoma after lumbar disk herniation. Ann Fr Anesth Reanim. 1997;16(3):307-9.

27. Mohanty A, Vasudev MK, Chandra PS. Extradural haematoma complicating lumbar puncture following a craniotomy. A case report. J Neurosurg Sci. 1998;42(4):233-7.

28. Scheil F, Larkamp U, Mutharoglu U. Spontaneous spinal epidural hematoma. Neurochirurgia (Stuttg). 1990;33(Suppl 1):45-9.

29. Ozdemir O, Calisaneller T, Yildirim E, Caner H, Altinors N. Acute spontaneous spinal subdural hematoma in a patient with bilateral incarcerated inguinal hernia. Joint Bone Spine. 2008;75(3):345-7.

\section{Endereço para correspondência}

José Luís Monteiro Alves

Serviço de Neurocirurgia de Coimbra,

Centro Hospitalar e Universitário de Coimbra

Praceta Prof. Mota Pinto

3000-075 - Coimbra, Portugal

Telefone: +35 19 1247-8316

E-mail: jlmonteiroalves@gmail.com 